\title{
Mycological Study of Dermatophytic Infections in and around Ambajogai, Maharashtra, India
}

\author{
Vidhya*, D.M. Kulkarni and S.L. Nilaker
}

Department of Microbiology, S.R.T.R Government Medical College, Ambajogai, India

*Corresponding author:

\begin{abstract}
A B S T R A C T
Dermatophytosis refers to superficial fungal infection of keratinized tissues, caused by group of keratinophilic dermatophytes. The prevalence of dermatophyte infection varies

\section{Keywords}

Dermatophytosis, Clinicomycological profile,

Tinea corporis, T.rubrum

Article Info

Accepted:

18 January 2019

Available Online:

10 February 2019 with different geographic area and climatic conditions. It is one of the common fungal infection but in recent times it is one of the neglected disease. The present study was aimed to assess the clinicomycological profile of dermatophytosis to identify the various infecting dermatophyte species and to compare clinical diagnosis with $\mathrm{KOH}$ microscopy and culture. A total of 198 clinically diagnosed cases of dermatophytosis were subjected to study during one year of. Specimens collected were skin scraping, nail clipping, and hair samples subjected to direct microscopy by $\mathrm{KOH}$ and fungal culture. During this study $\mathrm{KOH}$ microscopy revealed fungal elements in $(54.04 \%)$ cases while culture was positive in $(43.4 \%)$. Males were predominantly affected $(77.2 \%)$ as compared to female $(22.8 \%)$ and common age group affected was 21-30 years (38.2\%). Tinea corporis (50\%). was commonest clinical presentation. Trichophyton rubrum $(65.09 \%)$ was most common isolates followed by Trichophyton mentagrophytes (18.1\%). Among the dermatophytic skin infection, Tinea scorporis was the predominant clinical type and T. rubrum was most common isolate. Species level identification was necessary for epidemiological purpose and treatment.
\end{abstract}

\section{Introduction}

Despite the availability of effective antifungal agents, dermatophytic infections continue to be one of the principal dermatological diseases throughout the world ${ }^{(1)}$.The need for species identification of dermatophytes in clinical settings is of epidemiological concerns ${ }^{(1)}$. A skin infection due to dermatophytes has become a significant health problem affecting children, adolescents and adults (2). Dermatophytic infection is generally restricted to the nonliving cornified layer but a variety of changes occurs in the host because of the presence of the infectious agent and its metabolic products ${ }^{(2)}$.The type and severity of these reactions are related to the immune status of the host as well as to the strain and species of the organism causing the infection ${ }^{(2)}$. Dermatophytosis or ring worm infection or tinea is by far the most common disease in human beings ${ }^{(3)}$. Dermatophytic 
infections are hardly distinguishable clinically from each other, hence identification by in vitro culture is required for appropriate diagnosis and treatment as well as for epidemiological purpose.

At present, there are 42 species of dermatophytes classified into three genera Trichophyton, Microsporum and Epidermophyton $^{(4)}$. Clinical lesions caused by the fungi are highly variable and closely resemble other skin diseases making laboratory diagnosis and confirmation necessary $^{(5)}$.

So the present study was concerned with isolation, identification and clinicomycologial study of dermatophytes.

\section{Materials and Methods}

The current was study was conducted for the period of one year in the Department of Microbiology, S.R.T.R Government Medical College, Ambajogai. Clinically suspected 198 cases of dermatophytosis attending the outpatient Department were studied. First the details and history of the patients was recorded.

Skin specimens were collected by cleansing the affected area with $70 \% \mathrm{v} / \mathrm{v}$ ethanol and specimen were collected by scraping the surface of the margin of the lesion using a sterile blunt scalpel ${ }^{(6)}$. Nail pieces were collected by taking snipping of the infected part of the nail using sterile scissors. Where the nail was thickened scrapings from beneath the nail were also collected. ${ }^{(6,7)}$ Hairs were collect by removing dull broken hairs from the margin of the lesion using sterile tweezers or scraping the scalp with a blunt scalpel. Specimens were collected on clean piece of paper for transport to the laboratory ${ }^{.6,7)}$ Specimen collected was subjected to potassium-hydroxide $(\mathrm{KOH})$ wet preparation (10\% KOH for skin and hair; $40 \% \mathrm{KOH}$ for nail) for the presence of fungal elements. After direct microscopic examination, irrespective of demonstration of fungal elements, the specimen was inoculated on Sabouraud's dextrose agar with $0.05 \%$ chloramphenicol and $0.5 \%$ cycloheximide. This was incubated at $28^{\circ} \mathrm{C}$ for up to 4 weeks. If no growth was found after 4 weeks, culture was declared as negative. Mycological identification of the isolate was done based on macroscopic and microscopic examination. Any visible growth from SDA was examined for colony morphology, texture, surface pigmentation and pigmentation on reverse. Microscopic examination of colony was done by LCB mount to examine hyphal structure and arrangement of macroconidia and microconidia. Slide culture was done when there is difficulty in identifying fungal species. Other tests like hair perforation test, urease test were done to identify the isolate to species level ${ }^{(7)}$

\section{Results and Discussion}

Among the 198 clinically diagnosed cases of dermatophytosis, 153 (77.2\%) were males and $45(22.8 \%)$ were females. Majority of the patients belonged to the age group of 21-30 years (38.2\%), followed by 31-40 years $(16.9 \%)$ and $11-20$ years $(14.4 \%)$ (Fig. 1).

Among 198 specimens collected, 87\% were skin scrapings, $4.3 \%$ were hair samples and $8.7 \%$ were nail clippings.

Among total 198 samples, $\mathrm{KOH}$ mount reveled fungal element in 107(54.04\%) specimens, of these $78(39.5 \%)$ were culture positive and 29(14.54\%) were culture negative. Overall culture positive were $86(43.45 \%)$, among which 8(3.9\%) specimens were negative on $\mathrm{KOH}$ mount. 
Fig.1 Age wise distribution of clinical samples diagram of sex wise distribution
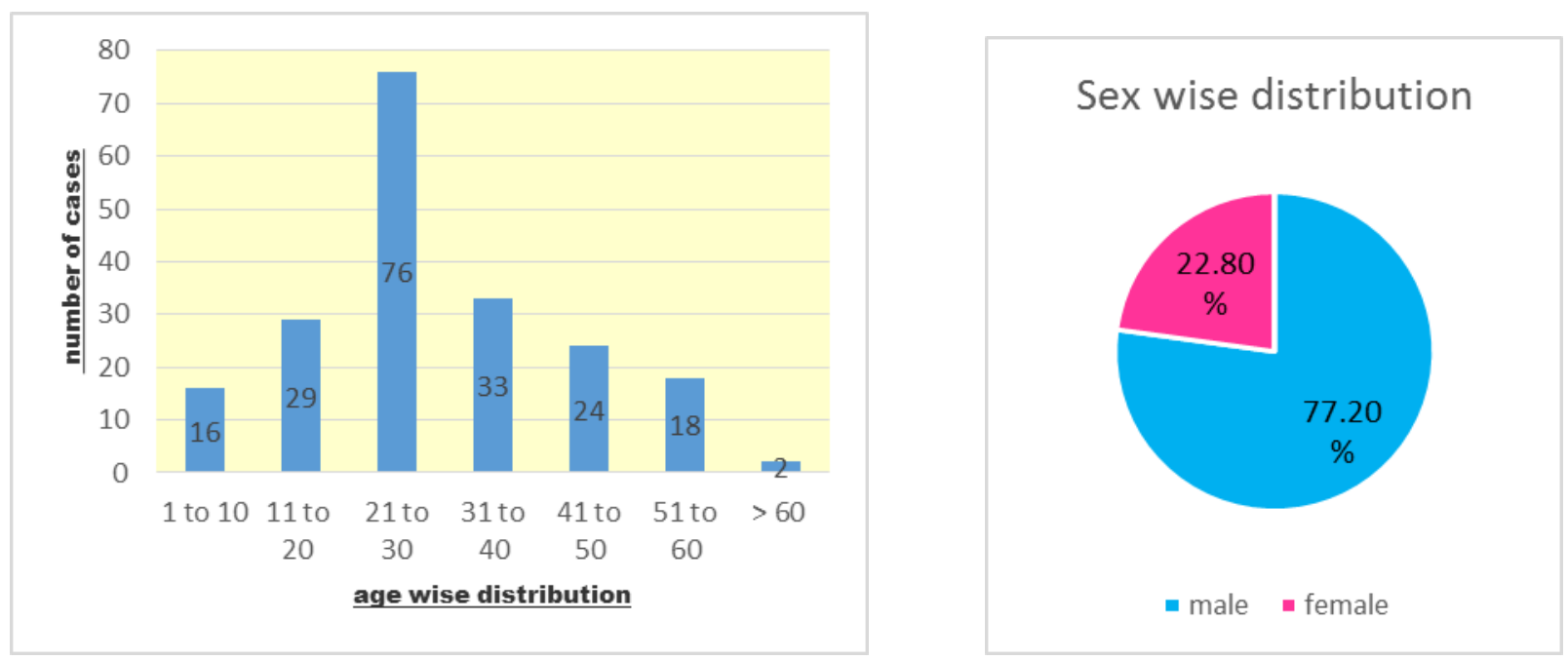

91 cases $(45.96 \%)$ were negative in both direct examination and culture. Among these $86(43.4 \%)$ culture positive isolates, 83 $(41.91 \%)$ were dermatophytes and $3(1.51 \%)$ were non dermatophytes. Trichophyton rubrum $56(65.09 \%)$ was the commonest isolate, followed by Trichophyton mentagrophytes $\quad 15(18.01 \%) \quad$ and Epidermophyton floccosum 5 (5.21\%), Trichophyton violaceum 4(4.91\%) and other non-dermatopyhtic isolates were 3 cases $(3.08 \%)$.

Tinea corporis was the predominant lesion in the present study occurring in $99(50.0 \%)$ patients followed by tinea cruris in 32 $(16.4 \%)$. Details are given in tables 1-3.

The mixed infection included a combination of tinea corporis and tinea cruris in $3(1.5 \%)$ patients

Dermatophytosis is a common disease in India like tropical countries and subtropical countries due to factors like heat and humidity. Morbidities of tinea infection are not only because of frequent relapses but also increasing resistance to antifungal drugs. The present study comprised of 198 clinically diagnosed cases of dermatophytosis.
Overall male predominance was observed which was consistent with other studies $(1,2,3,8)$. This can be correlated with the occupational hazards related to their nature of work, the frequent interaction with different people of the society, increased sweating in young males due to vigorous outdoor activity ${ }^{(2,9)}$. Higher incidence of dermatophytosis in our study was observed in $21-30$ years $(38.2 \%)$ and it was consistent with other studies ${ }^{(10)}$. The higher incidence of dermatophytosis in young age may be due to increased physical activity, opportunity for exposure and hormonal pattern ${ }^{(10,11)}$

Among total 198 samples, $\mathrm{KOH}$ mount reveled fungal element in 107(54.04\%) specimens, of these $78(39.5 \%)$ were culture positive and 29(14.54\%) were culture negative which was in correlation with other studies $(8,10,11)$. This $\mathrm{KOH}$ positivity and culture negativity could be due to nonviability of fungal elements in some cases ${ }^{(6)}$. In our study, overall culture positive were $86(43.45 \%)$, among which $8(3.9 \%)$ specimens were negative on $\mathrm{KOH}$ mount. 91 (42.06\%) did not show evidence of the fungi either on direct microscopy and culture. Nearly $8(3.9 \%)$ of the specimens were positive by culture alone and $29(14.54 \%)$ were by direct 
microscopy alone, highlighting the importance of both direct microscopy and culture in the definitive diagnosis of fungal infection.

In our study, Trichophyton rubrum were the commonest isolates (65.09\%) followed by Trichophyton mentagrophytes (18.01\%) which was consistent with other studies ${ }^{(10,11)}$.George ${ }^{[12]}$ has suggested that both the predominantly chronic nature of the infection and the adaptation of the dermatophyte to the human skin can explain the higher predominance of $T$. rubrum. In this study Epidermophyton floccosum were $5.21 \%$. It is closely similar with other studies ${ }^{(10,11)}$. Most of the isolates obtained were anthropophilic dermatophytes of Genus Trichophyton, and Epidermophyton which reflects the source of infection were probably from cases. In present study Trichophyton violaceum $(4.91 \%)$ and Trichophyton verrucosum (3.70\%).It was similar to other studies ${ }^{(8,10)}$. Trichophyton verrocosum is a zoophilic fungus that may signifies contact of people with domestic and pet animals, especially in rural area like ours.

Table.1 Comparison between $\mathrm{KOH}$ mount and culture positivity

\begin{tabular}{|l|c|c|c|}
\hline & $\begin{array}{c}\mathrm{KOH} \text { positive } \\
(\mathrm{n} \%)\end{array}$ & $\begin{array}{c}\mathrm{KOH} \text { negative } \\
(\mathrm{n} \%)\end{array}$ & Total $(\mathrm{n} \%)$ \\
\hline $\begin{array}{l}\text { Culture } \\
\text { positive }\end{array}$ & $78(39.5 \%)$ & $8(3.9 \%)$ & $86(43.4 \%)$ \\
\hline $\begin{array}{l}\text { Culture } \\
\text { negative }\end{array}$ & $29(14.54 \%)$ & $91(42.06 \%)$ & $112(56.6 \%)$ \\
\hline & $107(54.04 \%)$ & $91(45.96 \%)$ & $198(100 \%)$ \\
\hline
\end{tabular}

Table.2 Distribution of various species of dermatophytes

\begin{tabular}{|c|c|}
\hline Species & Percentage \\
\hline Trichophyton rubrum & $56(65.09 \%)$ \\
\hline Trichophyton mentagrophytes & $15(18.01 \%)$ \\
\hline Epidermophyton floccosum & $5(5.21 \%)$ \\
\hline Trichophyton violaceum & $4(4.91 \%)$ \\
\hline Trichophyton verrucosum & $3(3.70 \%)$ \\
\hline non dermatopyhtes & $3 \quad(3.01 \%)$ \\
\hline Total & $86(100 \%)$ \\
\hline
\end{tabular}

Table.3 Distribution of cases of dermatophytosis by clinical type

\begin{tabular}{|c|c|}
\hline CLINICAL TYPES & NUMBERS OF CASES \\
\hline Tinea corporis & $99(50 \%)$ \\
\hline Tinea cruris & $32(16.4 \%)$ \\
\hline Tinea capitis & $28(14 \%)$ \\
\hline Tinea unguium & $17(8.6 \%)$ \\
\hline Tinea pedis & $6(2.8 \%)$ \\
\hline Tinea faciei & $6(2.8 \%)$ \\
\hline Tinea manuum & $4(2.2 \%)$ \\
\hline Tinea barbae & $3(1.7 \%)$ \\
\hline Tinea corporis + Tinea cruris & $3(1.7 \%)$ \\
\hline Total & $198(100 \%)$ \\
\hline
\end{tabular}


Image.1\&2 Tinea corporis presentation \& Dermatophyte growth on SDA
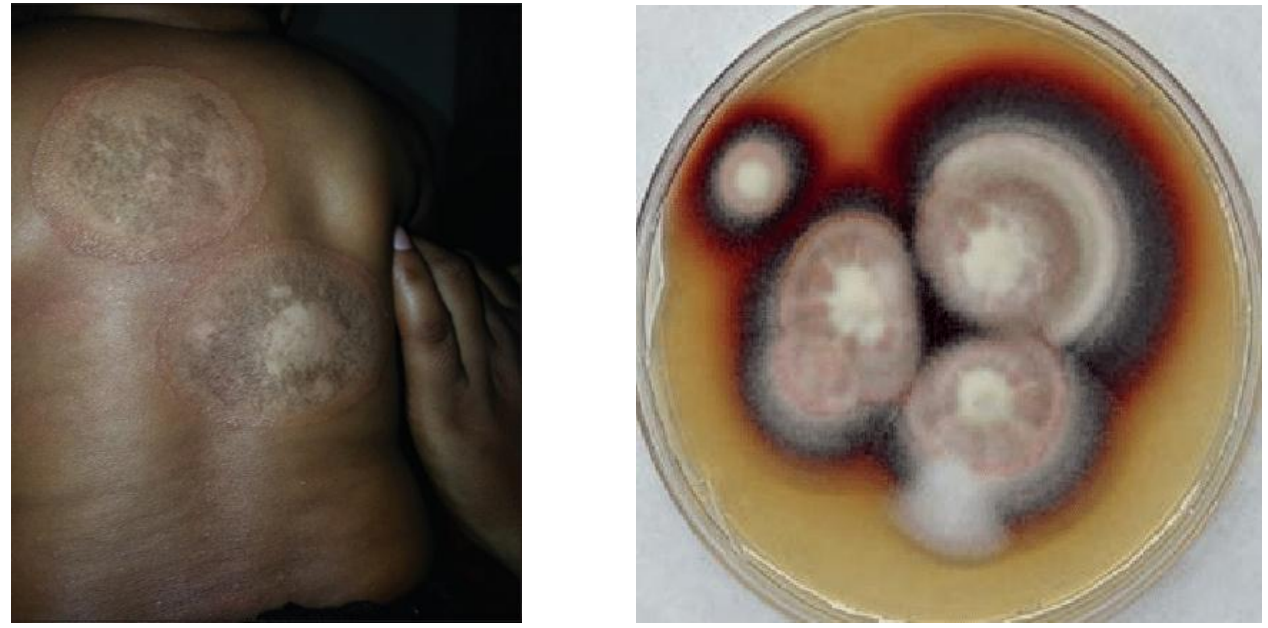

Image.3 showing fungal elements in $\mathrm{KOH}$ mount IMAGE 4- LCB mount of T. rubrum

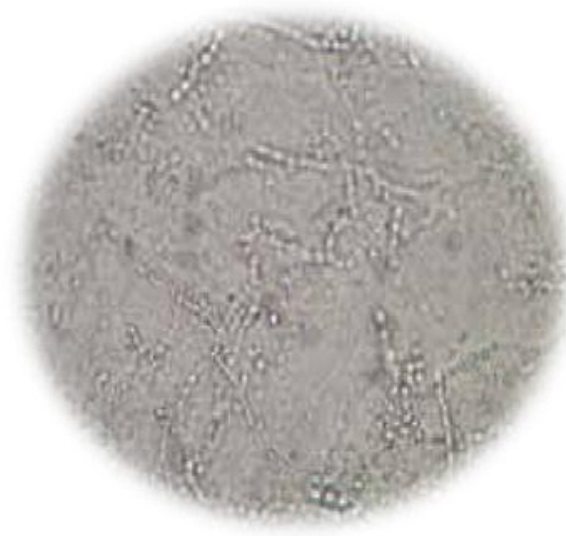

No Microsporum species were isolated in our study and it was consistent with other studies $(8,13)$. In the present study, other non dermatopyhtes were $3.08 \%$ among culture positive. It may be due to some other fungal infection that may produce dermatophytic like clinical lesion, it is indicating importance of culture.

The present study shows Tinea corporis were $(50 \%)$ as a commonest clinical presentation followed by Tinea cruris (16.4\%), Tinea capitis (14\%) which was conformity with other studies ${ }^{(1,11,14)}$. Less aeration due to tight clothing, maceration and high rate of sweating in groin area and waist region make this site more vulnerable to dermatophytosis ${ }^{(15)}$. The

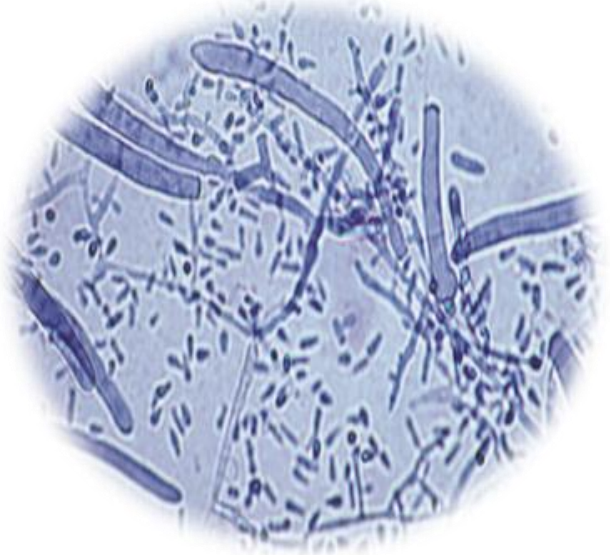

high incidence of tinea corporis and tinea cruris, due to its symptomatic nature (purities) which leads the patient to seek medical advice ${ }^{[16]}$. Tinea capitis was seen as predominant clinical type in children which may be due to poor scalp hygiene and relative neglect in hair dressing and sharing of fomites like towels, combs and lack of sebum ${ }^{(5)}$.

Our study showed Tinea unguium as $(8.6 \%)$, which was similar with other studies $(8,11)$ Our study showed $2.8 \%$ of Tinea pedis, $2.8 \%$ of Tinea faciea it is similar to other studies ${ }^{(15)}$. Mixed infection of tinea cruris and tinea corporis were observed only in negligible numbers and so also was tinea manuum and tinea barbae. 
In conclusion, because of contagious nature of dermatophytosis, an early diagnosis is needed. The present study highlights the use of both $\mathrm{KOH}$ and culture in the diagnosis of dermatophytosis for their effective and early treatment as it has psychological effect and a costly disease to treat. When either of these methods is used alone, it may result in false negative reporting. So it is important to perform species level identification for epidemiological purpose and also for treatment purpose because some species of dermatophytes show slower response to azole derivatives.

\section{References}

1.Mathur M, Kedia SK, Ghimire RBK. "“Epizoonosis of Dermatophytosis": A Clinico - Mycological Study of Dermatophytic Infections in Central Nepal." Kathmandu University medical Journal, 2012; 37(1): 30-3.

2.M.Jeya, G.Kumaran and. "Clinicomycological profile OF dermatophytic infections." Int J Pharm Bio Sci, 2014 April; 5 (2): (B) 1 - 5.

3.Madhavi S, Rama Rao MV and Jyothsna K. "Mycological study of Dermatophytosis in rural population." Scholars Research Library, 2011, 2 (3): 88-93.

4.Majeed N, Narayanankutty S, Rajan R, Theodore RB. Clinicomycological study of dermatophytosis in a tertiary care centre. J Acad Clin Microbiol [serial online] 2016 [cited 2018 Jul 12 ], and 18:110-113. n.d.

5.Janardhan, B., G. Vani. "Clinico mycological study of dermatophytosis." Int J Res Med Sci., 2017 Jan;5(1):31-39.

6.Cheesbrough, Monica. District Laboratory Practice in Tropical Countries Part 2. 2006.

7.Jagadish chander. Textbook of Medical Mycology: 4th Edition. 2018.
8.Tonita M. Noronha, Raghavendra S. Tophakhane. "Clinico-microbiological study of dermatophytosis in a tertiary-care hospital in North Karnataka." Indian Dermatol Online J, 2016;7:264-71.

9. Rashmi P Mahale, M Raghavendra Rao, Tejashree A, Deepashree R, Madhuri Kulkarni. "Clinicomycological profile of Dermatophytosis in a teaching hospital." International Journal of Pharmaceutical Science Invention, Volume 3 Issue 8; August 2014; PP.4346.

10.Sayeda Yasmeen Khadri,, Savita Koregol, Sayed Yunus Khadri, B.S. Patil. "Clinico-Mycological profile of Dermatophytes in Vijayapur, Karnataka." International Journal of Medical Microbiology and Tropical Diseases, April-June, 2016;2(2):75-78, n.d.

11.Sumit Kumar, P Shrikara Mallya, Pallavi Kumari. "Clinico-Mycological Study of Dermatophytosis in a Tertiary Care Hospital." International Journal of Scientific Study March 2014; Vol 1; Issue 6;27-32, n.d.

12.L George, K, Ann NY Acad Sci,1960, 89,69-99. n.d.

13. Poyyamozhi, J.S., Anandhi Lakshmanan. "Profile of dermatophyte infections among rural population: a facility based prospective observational study." International Journal of Community Medicine and Public Health, April 2018; Vol 5; Issue 4: 1354-1359.

14. Richa Sharma, Nakuleshwar Dut Jasuja, Suresh Sharma. "Clinical and mycological study of dermatophytosis in Jaipur." International Journal of Pharmacy and Pharmaceutical Sciences, Vol 4, Issue 3, 2012: 215-217. 15. Ghadage Dnyaneshwari P, Muley Vrishali A, Bhore Arvind V. "Clinicomycological Profile of 
Dermatophytosis in a Tertiary Care Hospital in Western India." SAS $J$. Med., Volume-1; Issue-4 (Nov-Dec, 2015); p-160-165, n.d.

16. Kennedy Kumar, Anupma Jyoti Kindo, J. Kalyani, S. Anandan. "Clinico - mycological profile of dermatophytic skin infections in a tertiary care center a cross sectional STUDY." Sri Ramachandra Journal of Medicine, January 2007;Vol.1; Issue 2;: 12-15.

\section{How to cite this article:}

Vidhya, D.M. Kulkarni and Nilaker, S.L. 2019. Mycological Study of Dermatophytic Infections in and around Ambajogai, Maharashtra, India. Int.J.Curr.Microbiol.App.Sci. 8(02):

2153-2159. doi: https://doi.org/10.20546/ijcmas.2019.802.249 